würde. Letzteres muss allerdings zeitlich beschränkt sein und auf die Wiederaufnahme normaler Beziehungen abzielen.

- Das Umgangsrecht kann auch geografisch beschränkt werden, indem es durch ein Ausreiseverbot ergänzt wird. Solche Maßnahmen dienen selbstverständlich der Vorbeugung internationaler Kindesentführungen. Der Richter ist befugt, ein Ausreiseverbot aus Frankreich (auch Übersee), das zeitlich unbegrenzt sein kann, insofern zu verhängen, dass beide Eltern der Ausreise ihre Einwilligung geben müssen. Die Staatsanwaltschaft lässt diese richterliche Entscheidung in die Datei für Personenfahndung eintragen.

- Das Umgangsrecht kann letztendlich bei Bedarf auch reduziert oder gar völlig aufgehoben werden. Dies kann z.B. bei Erkrankung oder Behinderung des Kindes oder eines Elternteils, bei Inhaftierung oder bei Verlust des Wohnsitzes der Fall sein.

\section{Der Beitrag zum Kindesunterhalt}

Streitigkeiten über den jeweiligen Beitrag zum Kindesunterhalt tragen tagtäglich zu einer wesentlichen Arbeitslast des Familienrichters bei. Um diese Last jedoch einzudämmen, ist immer häufiger die Rede von der Einführung einer Tabelle - etwa nach deutschem Muster - für die Bemessung des Unterhaltsbeitrags. Eine Tabelle ist bereits durch das Justizministerium veröffentlicht und zu Verfügung gestellt worden. Ihre Berücksichtigung durch die Parteien ist jedoch nicht verbindlich. Der Richter kann sie auch nicht in seiner Begründung benutzen, da seine Entscheidung ausschließlich auf Einkünfte und Ausgaben der Eltern und auf Kindesbedürfnisse gestützt werden darf.

\section{Schlusswort}

Sehr geehrte Kolleginnen und Kollegen, meine Damen und Herren,

gestatten Sie mir zum Schluss noch drei kurze Bemerkungen zu machen:

- Im Bereich des Familienrechts zielt die französische Gesetzgebung auf den Konsens, die Einvernehmlichkeit ab. Das Gesetz vom 4. März 2002 verleiht den Eltern die Möglichkeit, die Sorgerechtseinzelheiten selbst auszuwählen und zu gestalten. Der Richter kann je nach Ermessen dieser Wahl Rechtskraft verleihen.

- Unser gegenwärtiges Familienrecht ermöglicht erfreulicherweise die Erziehung des Kindes durch beide Eltern auch nach deren Scheidung und fördert somit eine ausgewogene affektive Bindung zwischen Eltern und Kind.

- Das gemeinsame Sorgerecht als Regelfall ist nun im französischen Familienrecht fest verankert. Ein zurzeit noch im Parlament zur Debatte stehender Gesetzentwurf sieht sogar vor, dass der Familienrichter befugt sein wird, eine Ordnungsstrafe von bis zu 10.000 Euro zu verhängen, wenn ein Elternteil dieser Regelung bewusst Hindernisse setzt oder gar gegen eine entsprechende richterliche Entscheidung verstoßen hat. Ich bedanke mich für Ihre Aufmerksamkeit und stehe selbstverständlich für jede Frage gerne zu Verfügung.

\title{
Elterliche Verantwortung in Deutschland: Verfahrensrechtliche Aspekte - Kindesanhörung und Mediation
}

\section{Elterliche Verantwortung in Deutschland}

1.

Um elterliche Verantwortung kann es zum einen im Verhältnis der Eltern zum Staat gehen.

Hier heißt es in Art. 6 Abs.2 GG: „Pflege und Erziehung der Kinder sind das natürliche Recht der Eltern und die zuvörderst ihnen obliegende Pflicht. Über ihre Betätigung wacht die staatliche Gemeinschaft."

Der Freiheitsraum der elterlichen Betätigung wird im Verhältnis zum Staat wie folgt abgegrenzt ${ }^{1}$ : Diese Normen garantieren den Vorrang der Eltern, ihre Eigenständigkeit und Selbstverantwortlichkeit bei der Pflege und Erziehung der Kinder, bestellen aber zugleich die staatliche Gemeinschaft zum Wächter. Das so umgrenzte Elternrecht [...] gewährt den Eltern ein Abwehrrecht gegen staatliche Eingriffe, soweit diese nicht durch das „Wächteramt“ gedeckt sind. Die Eltern haben das Recht, die Pflege und Erziehung ihrer Kinder nach ihren eigenen Vorstellungen frei zu gestalten und genießen Vorrang vor anderen Erziehungsträgern. Dieser Grundrechtsschutz darf aber nur für ein Handeln in Anspruch genommen werden, das bei weitester Anerkennung der Selbstverantwortlichkeit der Eltern noch als Pflege und Erziehung gewertet werden kann, nicht aber für das Gegenteil: die Vernachlässigung des Kindes. [...] Wenn Eltern in dieser Weise versagen, greift das Wächteramt des Staates nach Art. 6 Abs. 2 Satz 2 GG ein; der Staat ist nicht nur berechtigt, sondern auch verpflichtet, die Pflege und Erziehung des Kindes sicherzustellen. Diese Verpflichtung

1 BVerfG, Entscheidung v. 29.7.1968 - 1 BvL 20/63, 1 BvL 31/66 -, BVerfGE 24, 119 m.w.N.

\section{Ruth Feldkemper-}

Bentrup

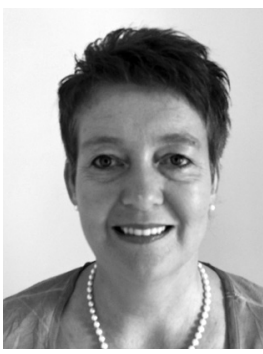

Richterin am

Oberlandesgericht Hamm, stellvertretende Vorsitzende des 11. Senats für Familiensachen 
des Staates folgt nicht allein aus dem legitimen Interesse der staatlichen Gemeinschaft an der Erziehung des Nachwuchses, aus sozialstaatlichen Erwägungen oder etwa aus allgemeinen Gesichtspunkten der öffentlichen Ordnung; sie ergibt sich in erster Linie daraus, dass das Kind als Grundrechtsträger selbst Anspruch auf den Schutz des Staates hat. Das Kind ist ein Wesen mit eigener Menschenwürde und dem eigenen Recht auf Entfaltung seiner Persönlichkeit im Sinne der Art. 1 Abs. 1 und Art. 2 Abs. 1 GG. [...] Die Anerkennung der Elternverantwortung und der damit verbundenen Rechte findet daher ihre Rechtfertigung darin, dass das Kind des Schutzes und der Hilfe bedarf, um sich zu einer eigenverantwortlichen Persönlichkeit innerhalb der sozialen Gemeinschaft zu entwickeln, wie sie dem Menschenbilde des Grundgesetzes entspricht. Hierüber muss der Staat wachen und notfalls das Kind, das sich noch nicht selbst zu schützen vermag, davor bewahren, dass seine Entwicklung durch einen Missbrauch der elterlichen Rechte oder eine Vernachlässigung Schaden leidet.

Diese Grundsätze hat das Bundesverfassungsgericht in zahlreichen seither ergangenen Entscheidungen stets wiederholt und in Einzelaspekten ergänzt. So hat es insbesondere auch darauf hingewiesen, dass es nicht zur Ausübung des Wächteramts des Staates gehört, gegen den Willen der Eltern für eine bestmögliche Förderung der Fähigkeiten des Kindes zu sorgen. Das Grundgesetz hat die primäre Entscheidungszuständigkeit von Eltern zur Förderung ihres Kindes anerkannt. Dabei wird auch in Kauf genommen, dass Kinder durch Entscheidungen der Eltern wirkliche oder vermeintliche Nachteile erleiden ${ }^{2}$.

\section{2.}

Nach deutschem Recht ist die elterliche Sorge wegen ihres Pflichtgehalts und wegen des Leitsatzes, dass Kontinuität in der Sorgebeziehung in der Regel dem Wohl des Kindes dient, in allen ihren Bestandteilen grundsätzlich unverzichtbar und - als höchstpersönliches Recht - unübertragbar.

Allerdings sieht das Gesetz in einigen Fällen Ausnahmen vor, in denen die Eltern mit einem übereinstimmenden Vorschlag, also einem kontrollierten Dispositionsakt, auf die Innehabung der elterlichen Sorge (teilweise) verzichten bzw. diese (teilweise) übertragen können, so z.B. im Fall des $\mathbb{\$} 1671$ Abs. 2 oder bei \1630 Abs. 3. Bei der Adoption wird die verfassungsrechtliche Elternstellung im Hinblick auf ein bestimmtes Kind übertragen; die elterliche Sorge endet bzw. „ruht“ mit der Einwilligung zur Adoption oder ihrer Ersetzung. Darüber hinaus kann die elterliche Sorge, insbes. die tatsächliche Personensorge, durch formlosen Vertrag der Ausübung nach übertragen werden (z.B. beim Dauer-Pflegevertrag oder bei der Internatserziehung). Derartige Abreden sind aber jederzeit frei widerruflich; entgegenstehende Vereinbarungen sind gem. $\mathbb{} 138$ Abs. 1 nichtig. ${ }^{3}$

\section{3.}

Elterliche Verantwortung betrifft vor allem das Verhältnis zwischen den Eltern und ihrem Kind/ihren Kindern. Die unserer Rechtsordnung zugrunde liegende Grundkonzeption der elterlichen Sorge ergibt sich aus $\mathbb{S} \mathbb{S} 1626,1627$ BGB.
\ 1626 Abs.2 BGB gibt ein Leitbild für die elterliche Erziehung vor. Die Pflichten, die den Eltern auferlegt werden, dienen dazu, das Kind schrittweise auf jene Selbständigkeit und jenes Verantwortungsbewusstsein vorzubereiten, die es bei Volljährigkeitseintritt erreicht haben soll. Soweit Fähigkeit und Bedürfnis der Kinder zu selbständigem verantwortungsbewusstem Handeln zu berücksichtigen sind oder soweit Argumenten des Kindes Rechnung zu tragen ist, tritt die elterliche Sorge zugunsten einer Anleitung des Kindes zur Eigenverantwortlichkeit zurück. Der Wandel der Intensität elterlicher Sorge stellt sich so als bewusste Selbstbeschränkung der Eltern zur Förderung der Persönlichkeitsentwicklung ihres Kindes dar. Mit $\mathbb{S} 1626$ Abs. 2 BGB ist ein über Jahrzehnte hinweg auf Gehorsam und Unterwerfung unter den Willen der Eltern ausgerichteter Erziehungsstil gesetzlich untersagt und durch eine partnerschaftliche Erziehung abgelöst worden. ${ }^{4}$

Die Berücksichtigung wachsender Fähigkeiten und Bedürfnisse des Kindes zu selbständigem verantwortungsbewusstem Handeln statuiert auch ein verfassungsrechtlich unbedenkliches formales Erziehungsziel. Wir haben eine Privatrechtsordnung, die jungen Menschen mit Vollendung des 18. Lebensjahres volle Geschäfts- und Deliktsfähigkeit zuweist. Das setzt notwendig das Erlernen der dazu erforderlichen Fähigkeiten durch schrittweise Einübung voraus. Die Erziehung des Minderjährigen zu einer eigenverantwortlichen Persönlichkeit entspricht dem in Art. 1, 2 GG zum Ausdruck kommenden Menschenbild des GG.

Rechtlich ist das so verstandene Sorgerecht auf allmähliche Verflüchtigung angelegt, darauf also, in allen Lebensbereichen, für die der Rechtsverkehr keine standardisierten Maßstäbe benötigt, im Einzelfall in bestimmten Schüben elterliche Fremdbestimmung durch Selbstbestimmung zu ersetzen. ${ }^{5}$

4.

Wenn Eltern sich trennen, stehen hinsichtlich ihres Kindes bzw. ihrer Kinder zahlreiche Entscheidungen an. In erster Linie müssen die Eltern den gewöhnlichen Aufenthaltsort und damit den Lebensmittelpunkt des Kindes bestimmen. Aber auch weitere Regelungen vor allem zum Umgang sind zu treffen oder müssen gegebenenfalls neu angepasst werden.

Obwohl Kinder die Trennung ihrer Eltern in der Regel nicht gewollt haben, sind sie in besonderer Weise davon betroffen. Ihr Wunsch, die Eltern mögen sich „für immer” lieben und „auf ewig” zusammenbleiben, geht nicht in Erfüllung. Durch das Scheitern der Paarbeziehung und die damit verbundene Neuorientierung der Eltern erhalten sie vorübergehend nicht die gewohnte Aufmerksamkeit und werden manchmal sogar in elterliche Konflikte hineingezogen. Sie haben Angst, einen Elternteil oder sogar beide Eltern zu verlieren, und suchen die Schuld für die Konflikte nicht selten bei sich selbst. Erst allmäh-

2 BVerfG, Stattgebender Kammerbeschluss v. 7.4.2014 - 1 BvR 3121/13 -, FamRZ 2014, 907 m.w.N.

3 Huber im Münchener Kommentar zum BGB, 6. Auflage 2012, § 1626 Rn $13 \mathrm{f}$.

Huber, a.a.O., § 1626 Rn 61.

5 Huber, a.a.O., § 1626 Rn 63 m.w.N. 
lich erlangen sie Gewissheit, dass elterliche Verantwortung die Trennung überdauern kann und muss.

In dieser Situation gehört es auch zur elterlichen Verantwortung im Sinne von $\mathbb{S} \mathbb{S} 1626$ Abs.2, 1627 BGB (s.a. Art. 12 der von den Vereinten Nationen 1989 verabschiedeten und von Deutschland 1992 ratifizierten UN-Kinderrechtskonvention), das betroffene Kind - seinem Alter und Verständnis entsprechend - an den anstehenden Entscheidungen zu beteiligen. Denn damit ist die Hoffnung verbunden, dass das Kind sich nach der Elterntrennung nicht erneut als Opfer einer Entwicklung erleben muss, auf die es keinen Einfluss nehmen kann. Beteiligung bringt das Kind in eine aktive Position, erhöht sein Verantwortungsbewusstsein und trägt dazu bei, dass seine Selbstwirksamkeit und sein Selbstwertgefühl gestärkt werden. Darüber hinaus lernt das Kind, dass Alternativen möglich sind, deren Vor- und Nachteile einer Abwägung bedürfen.

Auch die Eltern profitieren von der Partizipation des Kindes. Für sie verbindet sich hiermit eine größere Sicherheit, dass die Entscheidungen von allen Beteiligten getragen werden, auch bei auftretenden Konflikten tragfähig und dadurch nachhaltig sind. Sie erfahren mehr darüber, welche Gedanken sich das Kind über die veränderte Situation macht und dass es daraus eigene - bisweilen neuartige und für alle Gewinn bringende Vorschläge entwickelt. Nicht zuletzt machen die Eltern im Zuge der Beteiligung ihres Kindes die Erfahrung, trotz Trennung weiterhin als Eltern zu funktionieren und zu kooperieren.

Die Beteiligung des Kindes ist aber nicht grenzenlos. Kinder dürfen nicht unter dem Deckmantel der Partizipation für Zwecke der Eltern instrumentalisiert werden. Aufgabe der Eltern ist es vielmehr, ihre Kinder vor Überforderung zu schützen und sie davor zu bewahren, in für sie unlösbare Loyalitätskonflikte zu geraten. ${ }^{6}$

\section{Verfahrensrechtliche Aspekte}

\section{Orientierung am Kindeswohl - $\$ 1697$ a BGB:}

„Soweit nichts anderes bestimmt ist, trifft das Gericht in Verfahren über die in diesem Titel geregelten Angelegenheiten diejenige Entscheidung, die unter Berücksichtigung der tatsächlichen Gegebenheiten und Möglichkeiten sowie der berechtigten Interessen der Beteiligten dem Wohl des Kindes am besten entspricht.“

Es ist aber zu beobachten, dass sowohl der Vortrag von Eltern im gerichtlichen Verfahren, aber auch gerichtliche Entscheidungen zuweilen stark vom Gedanken der Sanktionierung eines Elternteils beeinflusst zu sein scheinen. Eine dem Elternrecht genügende Entscheidung kann aber nur aufgrund der Abwägung aller Umstände des Einzelfalls getroffen werden, bei der zu berücksichtigen ist, dass die Abwägung nicht an einer Sanktion des Fehlverhaltens eines Elternteils, sondern vorrangig am Kindeswohl zu orientieren ist. ${ }^{7}$ Das BVerfG (a.a.O.) hat wörtlich ausgeführt: „[...] offenbaren diese Formulierungen in kaum verhohlener Deutlichkeit, dass insoweit nicht das Kindeswohl, sondern die Sanktionierung der Beschwerdeführerin für angebliches Fehlverhalten im Vordergrund stand“.

Im Falle der Trennung oder Scheidung sind die Familienmitglieder in der Regel emotional hoch belastet. Nicht selten wird dadurch der Blick auf das Kindeswohl verstellt. Von den verfahrensrechtlichen Instrumenten, die dazu beitragen sollen, den Interessen des betroffenen Kindes Geltung zu verschaffen (z.B. Bestellung eines Verfahrensbeistands), sollen im Folgenden nur die Kindesanhörung und die Mediation näher betrachtet werden:

\section{Kindesanhörung}

\159 FamFG ist zunächst Ausdruck des verfassungsrechtlichen Gebots, in Kindschaftssachen den Willen des Kindes zu berücksichtigen, soweit dies mit seinem Wohl vereinbar ist. Voraussetzung hierfür ist, dass das Kind in dem gerichtlichen Verfahren die Möglichkeit erhält, seine persönlichen Beziehungen zu den Eltern erkennbar werden zu lassen. Die Gerichte müssen ihr Verfahren deshalb so gestalten, dass sie möglichst zuverlässig die Grundlage einer am Kindeswohl orientierten Entscheidung erkennen können. Mit der Kundgabe seines Willens macht das Kind zum einen von seinem Recht zur Selbstbestimmung Gebrauch. Denn jede gerichtliche Lösung eines Konflikts zwischen den Eltern, die sich auf die Zukunft des Kindes auswirkt, muss nicht nur auf das Wohl des Kindes ausgerichtet sein, sondern das Kind auch in seiner Individualität als Grundrechtsträger berücksichtigen, weil die sorgerechtliche Regelung entscheidenden Einfluss auf das weitere Leben des Kindes nimmt und es daher unmittelbar betrifft. Hat der unter diesem Aspekt gesehene Kindeswille bei einem Kleinkind noch eher geringes Gewicht, so kommt ihm im zunehmenden Alter des Kindes vermehrt Bedeutung zu. Ein vom Kind kundgetaner Wille kann Ausdruck von Bindungen zu einem Elternteil sein, die es geboten erscheinen lassen können, ihn in dieser Hinsicht zu berücksichtigen. Hat ein Kind zu einem Elternteil eine stärkere innere Beziehung entwickelt, so muss das bei der Sorgerechtsentscheidung berücksichtigt werden. ${ }^{8}$

$\int 159$ FamFG dient in Sorge- und Umgangsrechtsverfahren aber auch der Wahrung des verfahrensrechtlichen Gehalts von Art. 6 Abs. 2 S. 1 GG. Das Gebot zur persönlichen Anhörung, d.h. zur Anhörung des Kindes in der mündlichen Verhandlung durch das Gericht im jeweiligen Verfahren, erfüllt mehrere Funktionen:

Erstens wird dem Gericht ein persönlicher Eindruck von dem Kind vermittelt, der für die in Kindschaftssachen erforderliche Berücksichtigung des Kindeswohls ( $\mathbb{S} 1697$ a BGB) i.d.R. unerlässlich ist, zweitens wird dem Kind rechtliches Gehör gewährt und seine Stellung im Verfahren gestärkt und drittens wird dem Gebot der Sachaufklärung Genüge getan. Das Gericht kann insoweit durch Ausübung des richterlichen Fragerechts den Sachverhalt weiter aufklären, sich einen persönlichen Eindruck von den Beteiligten und deren Verhältnissen verschaffen und auf diese Weise seine Entscheidungsfindung optimieren. Daher kann auch eine Begutachtung des Kindes durch einen Sachverständigen die persönliche Anhörung durch den Richter nicht ersetzen. ${ }^{9}$

6 Vgl. Maywald, Die Beteiligung des Kindes an der Einigung der Eltern, FPR 2010, 460.

7 BVerfG, Beschluss v. 18.5.2009 - 1 BvR 142/09 -, FamRZ 2009, 1389.

8 BVerfG, Beschluss v. 18.5.2009 - 1 BvR 142/09 -, FamRZ 2009, 1389

9 Schumann im Münchener Kommentar zum FamFG, 2. Auflage 2013, § 159 Rn 1 m.w.N. 
Die Kindesanhörung soll nicht zuletzt dazu dienen, dass sich das Gericht ein Bild von den Bindungen des Kindes macht. Für die Feststellung des Vorliegens von Bindungen kann es auf beachtliche Gründe nicht ankommen. Denn gefühlsmäßige Bindungen können und brauchen nicht rational begründet $\mathrm{zu}$ werden, sie sind ein inneres Faktum. ${ }^{10}$

In diesem Zusammenhang wird häufig eingewendet, der - geäußerte - Kindeswille beruhe auf massiver Beeinflussung durch einen Elternteil, durch Großeltern oder Dritte, das Kind befinde sich in einem unauflösbaren Loyalitätskonflikt und deshalb sei der Kindeswille nicht oder jedenfalls nur eingeschränkt zu beachten. Hierbei könne es sich um das Phänomen der „illoyalen Bindung“ handeln, also um ein Eltern-Kind-Verhältnis, dessen Begründung oder Verstärkung auf pflichtwidrigem Verhalten eines Elternteils beruht, häufig zu beobachten bei Beeinflussung des Kindes gegen den anderen Elternteil. Es wird hinzugefügt, das, was das Kind äußere, sei einstudiert (Schallplatte), das Kind verdecke damit zugleich seine eigentliche seelische Haltung. Ob dies wirklich so ist, ist von Fall zu Fall zu ermitteln und häufig nur schwer festzustellen. An der Beachtlichkeit des geäußerten Kindeswillens ändert dies aber nichts. Denn auch eine illoyal begründete Bindung ist in der Person des Kindes ein Faktum, das im Interesse des Kindes nicht ignoriert werden darf. Auch bei erkannter oder doch zu vermutender Beeinflussung drücken Kinderwunsch und Kinderwille emotionale Bindungen aus. Auch durch Beeinflussung kann echte schützenswerte Bindung entstehen. Denn jede Erziehung ist Beeinflussung und keine persönliche Entscheidung ist unbeeinflusst, weder bei dem Kind noch bei einem Erwachsenen. Die Nichtbeachtung eines - insbesondere wiederholt - geäußerten Kindeswillens kann in diesem Zusammenhang nur in Betracht kommen, wenn feststeht, dass der geäußerte Kindeswille seine wirkliche Bindung nicht zutreffend wiedergibt.

Im Übrigen ist das Verhalten eines illoyalen Elternteils bei der Gesamtbeurteilung des Sachverhalts, insbesondere bei der Frage seiner Erziehungsfähigkeit, zu berücksichtigen. Es ist deshalb bedenklich, wenn Teile der Rechtsprechung etwa bei Umgangsablehnung durch ein Kind hierfür beachtliche oder verständliche Beweggründe verlangen, wie z.B. mehrfache Entführung oder das Bedürfnis des Kindes nach Ruhe und Stabilität. Solche Überlegungen machen deutlich, dass es der Rechtsprechung schwerfällt, bei der Ermittlung der Bindungen des Kindes von der den Erwachsenen geläufigen Rationalisierung abzusehen, die aber in diesem Zusammenhang keinen Raum hat. Die Frage nach vernünftigen Gründen gehört hier nicht zur Beachtlichkeit des Kindeswillens, sondern zur Prüfung des Kindeswohls im Übrigen und in dem Zusammenhang auch zur Berücksichtigung des Elternverhaltens. ${ }^{11}$

\section{Mediation}

\section{a) Mediationsgesetz vom 26. Juli 2012}

Wesentliches Ziel des Mediationsgesetzes (MediationsG)ist es, Verfahren der außergerichtlichen Konfliktbeilegung, insbesondere die Mediation, zu fördern. Hierbei kommt dem MediationsG zunächst die Funktion zu, dem Begriff der Mediation eine Kontur zu verleihen, einen rechtlichen Rahmen für die Durchführung einer Mediation zu schaffen und die Tätigkeit des Mediators im Interesse der Rechtspflege sowie zum Schutz der Medianden zu regulieren. Mit dem Gesetz sollen die Vorgaben der Richtlinie 2008/52/EG des Europäischen Parlaments und des Rates vom 21. Mai 2008 über bestimmte Aspekte der Mediation in Zivil- und Handelssachen (MedRL) umgesetzt werden. Seine Vorschriften sind daher richtlinienkonform auszulegen. ${ }^{12}$

Ohne hier auf Einzelheiten einzugehen, stößt die familienrechtliche Praxis auf das Problem, dass es für die außergerichtliche Mediation keine Verfahrenskostenhilfe gibt. Sie muss von den Beteiligten also selbst bezahlt werden. Ausnahmen gibt es allenfalls innerhalb wissenschaftlicher Forschungsvorhaben $(\mathbb{\$} 7$ Mediationsgesetz) oder bei Angeboten einzelner Jugendämter. ${ }^{13}$

\section{b) Güterichterverfahren gem. 36 Abs. 5 FamFG}

Als Ausfluss der allgemeinen Einigungsförderungspflicht kann das mit dem Verfahren befasste Gericht die Beteiligten in geeigneten Fällen nach $\mathbb{} 36$ Abs. 5 Gesetz über das Verfahren in Familiensachen und in den Angelegenheiten der freiwilligen Gerichtsbarkeit (FamFG) für einen Güteversuch vor einen nicht entscheidungsbefugten Richter verweisen. Geeignet sind Fälle, in denen aufgrund der Vorteile eines Güteversuchs vor einem Güterichter (z.B. konzentrierte Einigungsgespräche mit geringerem Zeitdruck, erhöhte Vertraulichkeit) unter Berücksichtigung des Gegenstands des Konflikts (z.B. Gestaltungsbefugnis) und der Person der Beteiligten (z.B. Bereitschaft zu Güteversuch) eine gewisse Wahrscheinlichkeit dafür besteht, dass das Verfahren infolge einer Erörterung vor dem Güterichter ohne streitige gerichtliche Entscheidung beendet werden kann. Güteversuche nach $\mathbb{3} 36$ Abs.5 FamFG sind nicht auf Streitverfahren beschränkt. Auch ist nicht zwingend nötig, dass die Beteiligten hinsichtlich des Verfahrensgegenstands dispositionsbefugt sind, weil eine gütliche Einigung nicht in einem (gerichtlich zu genehmigenden) Vergleich bestehen muss. Ausreichend ist, dass hinreichende Gestaltungschancen zur Auflösung eines zwischen mehreren Beteiligten bestehenden Konflikts gegeben sind (z.B. Verständigung über verfahrensrechtliche Fragen, Antragsrücknahme, Rücknahme eines Rechtsbehelfs o.ä.). Da eine Verständigung nach den Vorstellungen des Gesetzgebers nicht gegen den Willen der Beteiligten erzwungen werden soll, darf bereits die Verweisung nicht gegen den erklärten Willen der Beteiligten erfolgen. Die Verweisung ist nur für den Versuch einer gütlichen Einigung zulässig. Sie darf nur an einen Richter erfolgen, der zuvor im Geschäftsverteilungsplan generell-abstrakt hinreichend genau als Güterichter bestimmt wurde. ${ }^{14,15}$

10 Peschel-Gutzeit, Die Bedeutung des Kindeswillens, NZFam 2014, 433 m.w.N.

11 Peschel-Gutzeit, a.a.O., m.w.N.

12 Vgl. Ulrici im Münchener Kommentar zur ZPO, 4. Auflage 2013, Anhang zu § 278 a ZPO, Rn 1, 2.

13 Vgl. Greger, Mediation in Kindschaftssachen - Kosten, Akzeptanz, Nachhaltigkeit, FPR 2011, 117.

14 Ulrici im Münchener Kommentar zum FamFG, 2. Auflage 2013, §36 $\operatorname{Rn} 7,8$.

15 Zur praktischen Umsetzung der vergleichbaren Regelung des $\S$ 278 Abs.5 ZPO: Greger, Verweisung an den Güterichter und andere Formen konsensorientierter Prozessleitung, MDR 2014, 993. 


\section{c) Gerichtlicher Vorschlag der (außergerichtlichen) Mediation} gem. $\$ 36$ a FamFG

Die Vorschrift soll außergerichtliche Konfliktlösungsverfahren stärken, indem deren Durchführung - im Interesse der Beteiligten sowie zur Entlastung der Gerichte - auch noch nach Einleitung eines Verfahrens gefördert wird. Selbst in diesem Stadium ist es sinnvoll, den Beteiligten Alternativen zur autoritativen Entscheidung eines Konflikts durch einen Richter aufzuzeigen. Mit diesem Ziel wird zunächst die aus $\$ 36$ Abs. 1 S. 2 FamFG folgende und in jeder Lage eines Verfahrens bestehende gerichtliche Pflicht zur Förderung einer einvernehmlichen Konfliktlösung ausgestaltet. Neben der insoweit von $\$ 36$ Abs. 5 FamFG vorgesehenen Möglichkeit, die Beteiligten innerhalb des Verfahrens vor einen Güterichter zu verweisen, betont $\$ 36$ a Abs. 1 FamFG bei entsprechender Eignung als hierzu gleichwertige Möglichkeit die Mediation oder ein anderes von den Beteiligten einvernehmlich gewähltes außergerichtliches Konfliktlösungsverfahren. Lassen sich die Beteiligten auf ein solches Verfahren ein, setzt das Gericht gem. $\$ 36$ a Abs. 2 FamFG das Verfahren aus. $\$ 36$ a Abs. 3 FamFG stellt klar, dass außergerichtliche Konfliktlösungen nur unter Beachtung der der Dispositionsbefugnis der Beteiligten gesetzten Grenzen gefunden werden können. ${ }^{16}$

\section{d) Mediation in Kindschaftssachen}

Kennzeichnend für das Gerichtsverfahren sind: die Abgabe der Verantwortung an das Gericht, ein nach im Wesentlichen zwingenden Regeln ablaufender Prozess, der Abschluss des Verfahrens durch einen juristisch fundierten Vergleich oder eine Gerichtsentscheidung und die Kommunikationsform des logischen Argumentierens. ${ }^{17}$

Bei Streitigkeiten um das elterliche Sorge- und Umgangsrecht geht es aber oftmals nicht um Rechtsanwendung, sondern um die Gestaltung zwischenmenschlicher Beziehungen, die sich an den Bedürfnissen und Werten der daran Beteiligten (Kinder, Mutter, Vater) orientiert. Niemand ist dazu besser in der Lage als die Betroffenen selbst. Diese Konflikte scheinen somit prädestiniert zu sein für Mediation. Jeder verantwortungsbewusste Anwalt, der mit einem elterlichen Konflikt um Kinder betraut wird, sollte zumindest ernsthaft in Erwägung ziehen, dem von ihm vertretenen Elternteil zu raten, statt der streitigen Auseinandersetzung vor Gericht die einvernehmliche Lösung im Gespräch miteinander, gegebenenfalls angeleitet durch einen Berater oder Mediator, zu suchen. ${ }^{18}$

Bei der Mediation sind der materielle und der verfahrenstechnische Aspekt zu unterscheiden. ${ }^{19}$ Zum Ersten sind da die Eckpfeiler der Mediation, wie die Eigenverantwortung der Medianden für die Lösung ihres Konfliktes. Zum Zweiten ist eine Mediation ein sich in Schritten vollziehender Prozess mit dispositiven Regeln. Im Zentrum einer jeden Mediation steht der Übergang von den Positionen zu den dahinter liegenden Interessen und Bedürfnissen der am Konflikt beteiligten Parteien, aus denen sich die Lösung des Konfliktes ableitet. Schließlich ist Mediation ein spezieller Kommunikationsprozess mit Kommunikationswerkzeugen, beispielsweise das Reframing (in einen anderen Rahmen setzen $=$ Verändern des Blickwinkels).
Die enorm hohe Anzahl an gerichtlichen Sorge- und Umgangsrechtsverfahren zeigt, dass der Weg der Mediation nur selten beschritten wird. In vielen Fällen ist die Beziehung zwischen den Eltern derart emotional belastet, dass sie sich zu einer konstruktiven Konfliktlösung nicht mehr in der Lage fühlen. Mitunter stehen auch Gewalt- oder Missbrauchsszenarien, Suchtprobleme oder psychische Erkrankungen einer solchen Lösung im Wege. Häufig liegt es aber einfach an Unwissenheit und Fehlvorstellungen, dass selbst vernünftige Eltern den - je nach Lage des Falles - unnötigen Weg zum Gericht einschlagen (und - wenn es gut geht - dort zu einer Einigung finden). Auch Anwälte gehen oft davon aus, dass sie ihrem Mandanten nicht den ungewissen und kostspieligen Gang in die Mediation empfehlen dürfen, wenn das gerichtliche Verfahren auf jeden Fall eine (einvernehmliche oder rechtskonforme) Lösung zu berechenbaren Kosten, bei Verfahrenskostenhilfe sogar kostenfrei, bietet. Dieser gedankliche Ansatz greift aber möglicherweise zu kurz. Gerade in Sorgerechtsstreitigkeiten entstehen im gerichtlichen Verfahren oft hohe Kosten durch Sachverständigengutachten, die - vom Grundsatz her - auch von den Beteiligten selbst zu tragen sind. ${ }^{20}$

Eine vom Bundesjustizministerium initiierte und von den Landesjustizverwaltungen unterstützte Pilotstudie, die in den Jahren 2007 bis 2010 erstellt wurde, ergab in Fällen der Mediation eine hohe Verfahrenszufriedenheit bei den Eltern. ${ }^{21}$ Das Ziel der Mediation, eine den Interessen beider Seiten gerecht werdende Lösung zu finden, wurde also in aller Regel erreicht. Auch die Beratungsanwälte berichteten von einer hohen, beiderseitigen Zufriedenheit, auch noch nach längerer Zeit. Hingegen berichteten die Eltern in fast der Hälfte der Fälle der Gerichtsverfahren, dass sich die gerichtliche Entscheidung auf ihre Beziehung zueinander negativ ausgewirkt habe. Auch die Entwicklung der Eltern-Kind-Beziehung wurde negativ bewertet.

Greger (a.a.O.) weist auf die geradezu alarmierenden Antworten der Eltern auf die Frage hin, wie sich die Entscheidung auf die Entwicklung der Kinder ausgewirkt habe. Auch wenn die fehlende Repräsentativität der Ergebnisse in Rechnung gestellt werde, müsse es zu denken geben, wie häufig Eltern berichteten, dass ihre Kinder unter der getroffenen Regelung litten, Verhaltensauffälligkeiten oder Entwicklungsstörungen zeigten oder sich in therapeutischer Behandlung befänden. Insbesondere die Entscheidungen zum Umgang mit dem nicht sorgeberechtigten Elternteil riefen bei den Kindern offenbar große Belastungen hervor: Nur in geringem Umfang würden diese Entscheidungen nach Einschätzung der Eltern von den Kindern akzeptiert. Die meisten arrangierten sich, hätten aber lieber eine andere

16 Vgl. Ulrici im Münchener Kommentar zum FamFG, 2. Auflage 2013, $\S 36$ a Rn 1.

17 Spangenberg/Spangenberg, Hinwirken auf Einvernehmen als kommunikativer Prozess, FPR 2013, 460.

18 Vgl. Greger, Mediation in Kindschaftssachen-Kosten, Akzeptanz, Nachhaltigkeit, FPR 2011, 115.

19 Vgl. Spangenberg/Spangenberg, a.a.O.

20 Vgl. Greger, a.a.o.

21 Greger, a.a.o. 
Regelung. Jedes fünfte Kind leide unter der Regelung, etwa in derselben Größenordnung würden Verhaltensauffälligkeiten, Entwicklungsstörungen und Therapiebedarf gemeldet. Aufschlussreich sei auch, dass vielfach auch die Anwälte, die Eltern in streitigen Umgangs- oder Sorgerechtsverfahren vertreten haben, nicht davon überzeugt seien, dass sich die getroffene Entscheidung zum Wohl des Kindes auswirken werde.

Von den Eltern, die ihren Konflikt im Wege der Mediation beigelegt haben, würden derartige Auswirkungen kaum berichtet. Die Entwicklung der Eltern-Kind-Beziehung sei - in Bezug auf beide Elternteile - nur in wenigen Ausnahmefällen negativ beurteilt worden. Die Beziehung zwischen den Eltern habe sich laut der Hälfte der Berichte durch die Mediation verbessert, also genau konträr zur Situation bei den Gerichtsverfahren entwickelt. In Bezug auf die Entwicklung der Kinder würden negative Auswirkungen ebenfalls nur in wenigen Einzelfällen berichtet. Dies bestätige die wiederholt von den Praktikern geäußerte Erfahrung, dass Kinder, die einen konstruktiven Umgang der Eltern mit der Trennungssituation erleben, diese ebenfalls besser bewältigen können. ${ }^{22}$
Aus diesem für die Qualität der Mediation sprechenden Ergebnis sollten keine Schlüsse zu Lasten des Gerichtsverfahrens gezogen werden. Zutreffend dürfte aber der (umgekehrte) Schluss auf die Qualität der Mediation sein: Wenn sie zu einer einvernehmlichen Lösung des Elternkonflikts führt, zeichnet sich diese in der Regel durch hohe Akzeptanz und Nachhaltigkeit aus. Negative Auswirkungen auf die Beziehung zwischen den Beteiligten und auf die Entwicklung des Kindes sind wesentlich seltener, wenn die Eltern die Lösung ihres Konflikts nicht streitig vor Gericht, sondern im Wege der Mediation suchen. Die o.g. Untersuchung hat aufgezeigt, dass bei vielen vom Richter zu beurteilenden Fällen schon während des Verfahrens massive Persönlichkeits- und Beziehungsstörungen vorlagen. Wenn in solchen Fällen oder bei völlig inkooperativem Verhalten der Eltern eine richterliche Entscheidung getroffen werden muss, können deren schädliche Auswirkungen nicht auf Defizite des gerichtlichen Verfahrens zurückgeführt werden. ${ }^{23}$

23 Greger, a

\section{Entwicklung der elterlichen Verantwortung im europäischen Kontext unter besonderer Berücksichtigung von Trennung/Scheidung und Genderaspekten}

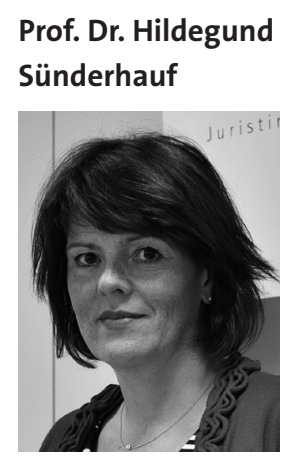

Professorin für Familienrecht und Kinder- und Jugendhilferecht an der Evangelischen Hochschule Nürnberg/Autorin der Monografie: Wechselmodell: Psychologie-Recht Praxis, Wiesbaden, Springer VS (2013)
1. Elterliche Verantwortung in Europa

1.1. Begriff und Bedeutung der elterlichen Verantwortung

Elterliche Verantwortung ist ein Oberbegriff für die Bereiche

- Inhaberschaft und Inhalte der elterlichen Sorge (\$S 1626, 1626a, 1671, 1672 BGB)

- Entscheidungsbefugnisse im Rahmen der elterlichen Sorge $(\mathbb{S} 1687$ bis $1687 \mathrm{~b}$ BGB)

- Umgangsrecht und Umgangspflicht $(\mathbb{S} \mathbb{S} 1684-$ 1686 BGB) und

- Kindesunterhalt, als Ausdruck der ökonomischen elterlichen Verantwortung ( $\mathbb{S} 1601 \mathrm{ff}$. BGB), insbesondere auch die Gleichwertigkeit von Barunterhalt und Betreuung ( $\mathbb{S} 1606$ Abs. 3 Satz 2 BGB). Der folgende Beitrag beschränkt sich auf die Bereiche elterliche Sorge und Umgangsrecht. Im Rahmen des Sorge- und Umgangsrechts ist elterliche Verantwortung im BGB näher umrissen durch die Verpflichtung der Eltern,

- ihre Entscheidungen unter altersgerechter Einbeziehung des Kindes zu treffen ( $\$ 1626$ Abs. 2 BGB)

- ihre Entscheidungen und ihr Handeln stets am Kindeswohl auszurichten ( $\mathbb{1 6 2 7}$ Satz 1 BGB),
- sich in Kindesbelangen um Einigung zu bemühen ( $\int 1627$ Satz 2 BGB), was auch die Pflicht beinhaltet, eine positive Kooperation und Kommunikation mit dem anderen Elternteil zu suchen und

- die Bedeutung des anderen Elternteils als gleichwertige, ebenfalls wichtige Bezugsperson des Kindes anzuerkennen, die zu dessen Persönlichkeitsentwicklung von grundlegender Bedeutung ist, die sog. Bindungstoleranz ( $\mathbb{S} 1626$ Abs. 3 Satz 1, 1684 Abs. 1 und 2 Satz 1 BGB).

Zentrale Bedeutung hat die elterliche Verantwortung natürlich in der grundrechtlich geschützten pädagogische Erziehungsverantwortung beider Eltern (Art. 6 Abs. 2 GG „Pflege und Erziehung der Kinder sind das natürliche Recht der Eltern [...].“) und zwar unabhängig von der rechtlichen Sorgerechtslage und vom Betreuungsmodell. Dies spiegelt sich u.a. in der sog. Wohlverhaltenspflicht der Eltern in $\$ 1684$ Abs. 2 BGB wider, wonach Eltern im Rahmen von Umgangskontakten alles zu unterlassen haben, „was das Verhältnis des Kindes zum jeweils anderen Elternteil beeinträchtigt oder die Erziehung erschwert". Schon aus dem Wortlaut der Norm lässt sich schließen, dass auch im Getrenntleben beide Eltern an der Erziehung eines Kindes beteiligt sind, denn die 